\title{
Evaluation of the potential in radiation dose reduction for full-field digital mammography
}

\author{
${ }^{1}$ University of Applied Sciences (TFH), Berlin, Germany \\ ${ }^{2}$ King Faisal Specialist Hospital and Research Center, Jeddah, Saudi-Arabia \\ e-mail: kasch@tfh-berlin.de
}

\begin{abstract}
This study evaluates the image quality for different radiation doses in full-field digital mammography (FFDM). The potential of dose reductions is evaluated for both, the transition from screen-film mammography (SFM) to FFDM as well as within FFDM due to the optimization of exposure parameters.
\end{abstract}

Exposures of a $4.5 \mathrm{~cm}$ breast phantom rendering different contrasts as well as bar patterns were made using a FFDM system (GE Senographe 2000D). For different kVp and mAs settings as well as different target/filter combinations chosen for the above exposures, average glandular dose (AGD), signal-to-noise ratio (SNR), contrast-to-noise ratio (CNR) and modulation transfer function (MTF) were determined. To benchmark the results, relative change of AGD was evaluated against SNR, CNR and MTF. Eventually, the results were normalized to AGD's rendered by settings typically used in today's clinical routine.

For standard settings (automatic mode), both FFDM and SFM deliver approximately the same AGD of about $2.2 \mathrm{mGy}$. From that, AGD reduction can be substantial in FFDM if only SNR and high contrast CNR are considered. In this case, reduction of up to $40 \%$ can be achieved in a wide $\mathrm{kVp}$ range if switching from the standard target/filter combination $\mathrm{Mo} / \mathrm{Rh}$ to $\mathrm{Rh} / \mathrm{Rh}$. However, if low contrast $\mathrm{CNR}$ is to remain unchanged, dose reduction is practically impossible. The change of peak voltage and target/filter material had no influence on MTF.

Assuming current CNR requirements as standards, significant dose reduction in FFDM cannot be achieved. Only by compromising low contrast CNR levels AGD of up to $40 \%$ can be saved at current standards of SNR and high contrast CNR.

Key words: Conventional mammography, digital mammography, contrast, dose reduction. 


\section{Introduction}

There has been a lot of debate about dose reduction in digital mammography compared to conventional screen-film mammography (SFM) [3, 4, 6]. The main argument for the reduction potential in full-field-digital mammography (FFDM) is the high dynamic range of the detector and its linear relationship between dose and signal intensity as opposed to the sigmoid relationship between optical density and dose in screen-film systems. Furthermore, digital systems offer image post-processing that can be used to elicit even more information from the digital image.

As of today, however, most installations of FFDM seem to operate at settings rendering about the same doses as state-of-the-art SFM [3, 6]. This is most likely due to the fact, that clinical studies concerning dose reduction in FFDM are rare and, if they exist, warn about potential losses of clinical information such as microcalcification detectability [4].

Image quality in mammograms is a trade-off between spatial and contrast resolution. While spatial resolution in FFDM is limited by detector resolution rather than by noise, i.e. dose, contrast resolution will decide the degree of possible dose reduction. While most dose reduction studies evaluate the final information after image acquisition and image processing, this study aims at the following question: What is the dose reduction potential if the currently used contrast (and spatial) resolution levels are to be kept? This approach tests the (assumedly advantageous) response of the digital detector, i.e. it evaluates the potential dose reduction without employing any image enhancements during image post processing.

\section{Materials and Methods}

The basic data to which all relative dose change is referenced were taken from our quality assurance measurements at a Mammomat 1000 (Siemens) and compared to other SFM results given in the literature $[2,5]$. Our results, averaged over the clinically used settings, render $2.2 \mathrm{mGy}$ average glandular dose (AGD) for a $4.5 \mathrm{~cm}$ thick breast (phantom) simulating 50/50 glandular/fatty breast tissue and are in accordance with the values given for comprehensive surveys [cf. 2, 5].

Also from our quality assurance measurements we gather a $2.2 \mathrm{mGy}$ (AGD) for the same $4.5 \mathrm{~cm}$ thick phantom at the FFDM system Senographe 2000D (GE Medical 
Systems) operated at AOP (automatic operating parameter) mode STD (standard) where it utilizes a Mo/Rh target filter combination at $28 \mathrm{kVp}$.

All measurements were then performed at the Senographe 2000D at standard Focus-Detector-Distance (FDD). The phantom used was a $4.5 \mathrm{~cm}$ thick breast phantom simulating 50/50 glandular/fatty breast tissue. It features a high contrast insert $(2.5 \mathrm{~mm}$ Teflon) and several drilled holes of 0.1 through to $0.4 \mathrm{~mm}$ depth rendering low contrast areas.

Measurements in steps of $2 \mathrm{kVp}$ were taken for all three target/filter combinations, i.e. $\mathrm{Mo} / \mathrm{Mo}, \mathrm{Mo} / \mathrm{Rh}, \mathrm{Rh} / \mathrm{Rh}$. The peak voltage ranges in $\mathrm{kVp}$ for each combination were $26 / 34,28 / 40$, and $31 / 45$, respectively. For each target/filter combination at each voltage three mAs settings were used: 50, 100, and the highest tolerated by the tube (e.g. 280 $\mathrm{mAs}$ at $31 \mathrm{kVp}$ for $\mathrm{Rh} / \mathrm{Rh})$. These 60 images were saved for further processing.

The Senographe 2000D features a build-in algorithm giving an estimate of the Entrance Skin Dose (ESD) at standard FDD based on the exposure parameter set and the compression thickness detected. From that, using conversion tables taken from [6] the system also gives the AGD corresponding to that ESD. Thus, ESD and AGD were recorded along the above set of measurements.

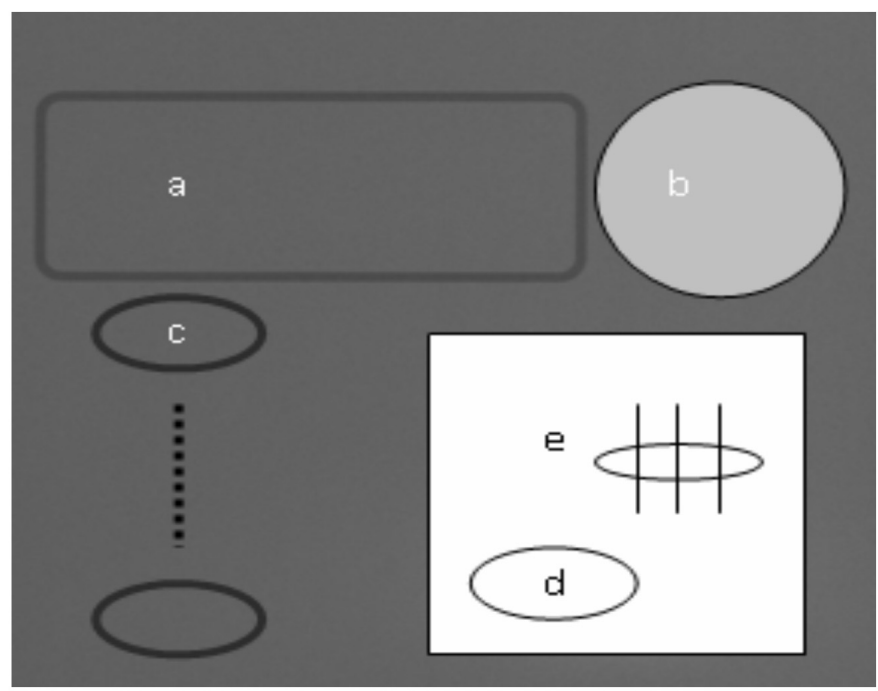

Figure 1. Schematic representation of the regions of interest (ROI) that were evaluated as described in the text. The drawing is not to scale 
The evaluation software of the Senographe's acquisition workstation (AWS) was utilized to delineate the following regions of interest (ROI) in each raw (i.e. unprocessed) phantom image (cf. Figure 1):

a) background (bg) signal,

b) high contrast (cnt) signal,

c) low contrast (cnt) signal (as many as visible, maximum 7),

d) bar pattern attenuation (bpa) signal,

e) individual bar pattern (bp) signal (0.6-5) lp/mm.

All ROI's included at least 1000 pixel. Using the AWS software (operating on a 14 bit deep gray scale) mean pixel value $\mathrm{M}$ and standard deviation $\sigma$ were recorded for each ROI; except for (d) and (e) where only M and $\sigma$ was recorded, respectively.

The parameters signal-to-noise ratio (SNR), contrast-to-noise ratio (CNR) and modulation transfer function (MTF) are then calculated as follows:

$$
\begin{gathered}
\mathrm{SNR}=\frac{M}{\sigma} \\
\mathrm{CNR}=\frac{\left|M_{b g}-M_{c n t}\right|}{\sigma_{b g}}=\mathrm{SNR}_{b g}\left|1-\frac{M_{c n t}}{M_{b g}}\right| \\
\mathrm{MTF}=\frac{2.2 \sigma_{b g}}{\left|M_{b g}-M_{b p a}\right|}
\end{gathered}
$$

The definitions in Equations (1) through (3) secure the independence of the parameter from any windowing and levelling changes due to image post processing. A derivation for the MTF according to Equation (3) is given in the appendix.

For benchmarking the results three ratios are evaluated:

$$
\begin{aligned}
& \mathrm{AGD}_{\mathrm{SNR}}=\frac{\mathrm{AGD}}{\mathrm{SNR}^{2}} \\
& \mathrm{AGD}_{\mathrm{CNR}}=\frac{\mathrm{AGD}}{\mathrm{CNR}^{2}}
\end{aligned}
$$




$$
\mathrm{AGD}_{\mathrm{SNR} C \mathrm{CNR}}=\frac{\mathrm{AGD}}{\mathrm{SNR} \cdot \mathrm{CNR}}
$$

Since SNR is proportional to the square root of dose and dose is proportional to mAs if peak voltage and target/filter combination are constant, $A G D_{S N R}$ in equation (4) is only a function of $\mathrm{kVp}$ and target filter combination. The same holds true for equations (5) and (6) since the data show $M_{c n t}$ and $M_{b g}$ from equation (2) in a first approximation to be proportional to dose (i.e. $\mathrm{mAs}$ ) for constant $\mathrm{kVp}$ and target filter combination. Therefore, their ratio is also a function of only $\mathrm{kVp}$ and target filter combination.

All three ratios were determined for all phantom images and, for each image, for all contrast steps that could be evaluated. They were then averaged over the three mAs values taken for each peak voltage at each target/filter combination. Eventually, the ratios were normalized to $28 \mathrm{kVp}$ for the $\mathrm{Mo} / \mathrm{Rh}$ target/filter combination and plotted as a function of peak voltage and target filter combination.

\section{Results}

Figures 2 through 5 summarize the results showing the relative breast dose (AGD) as a function of peak voltage and target/filter combination for a constant SNR, CNR and SNR/CNR product; the latter two for high contrast and low contrast conditions. All graphs are normalized on $28 \mathrm{kVp}$ for a Mo/Rh target/filter combination. The obvious interpretation of the graphs is, that they show the relative change of dose (hence AGD) to be given compared to the standard $\mathrm{kVp}$ and target/filter setting under the assumption, that the SNR, CNR, or product of SNR and CNR remain unchanged.

The MTF according to equation (3) turned out to be almost invariant to the change in acquisition parameters. Figure 7 shows a sample MTF for $\mathrm{Rh} / \mathrm{Rh}$ target filter combination at $45 \mathrm{kVp}$. With all MTF measured being the same within the statistical fluctuations figure 8 gives the MTF values at $2.2 \mathrm{lp} / \mathrm{mm}, 3.1 \mathrm{lp} / \mathrm{mm}$, and $5.0 \mathrm{lp} / \mathrm{mm}$ for all measured target filter combinations and peak voltages.

\section{Conclusion}

The results show both dose reduction options as well as their clear limits utilizing a Senographe 2000D as FFDM system. 


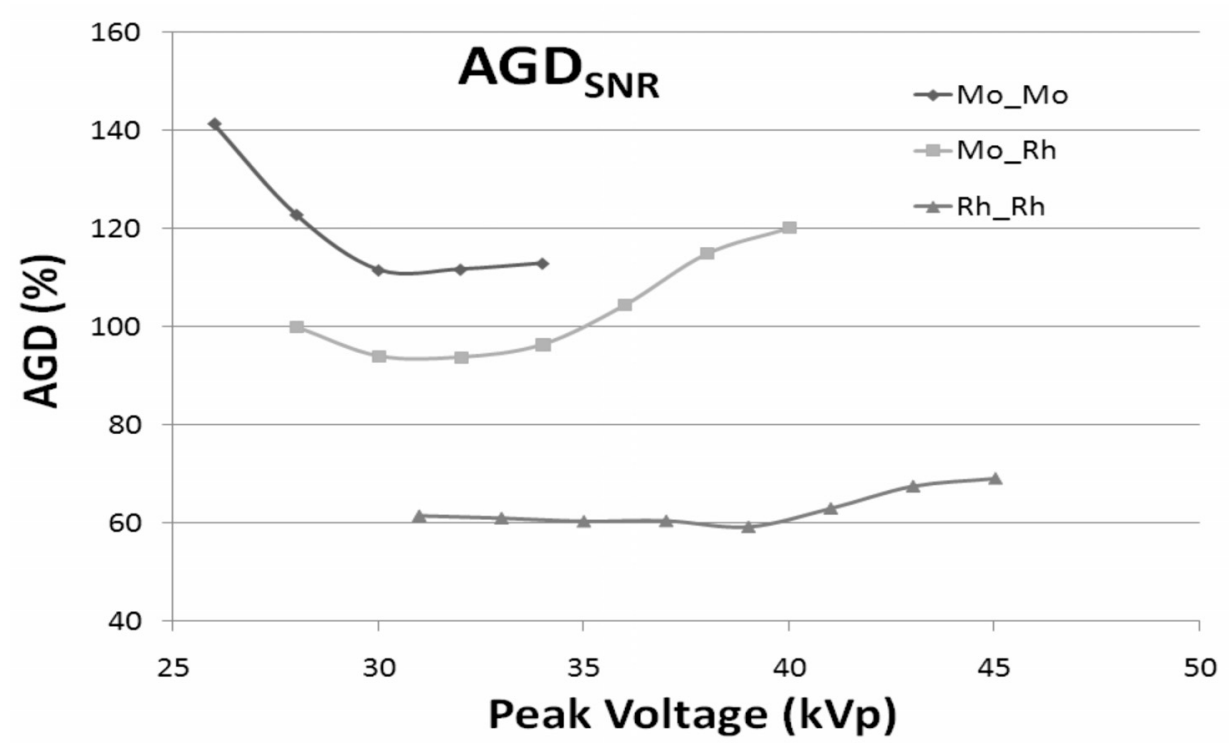

Figure 2. Relative AGD if SNR is to remain constant for different $k V p$ and target filter combinations. The graphs are normalized to $28 \mathrm{kVp}$ for $\mathrm{Mo} / \mathrm{Rh}$

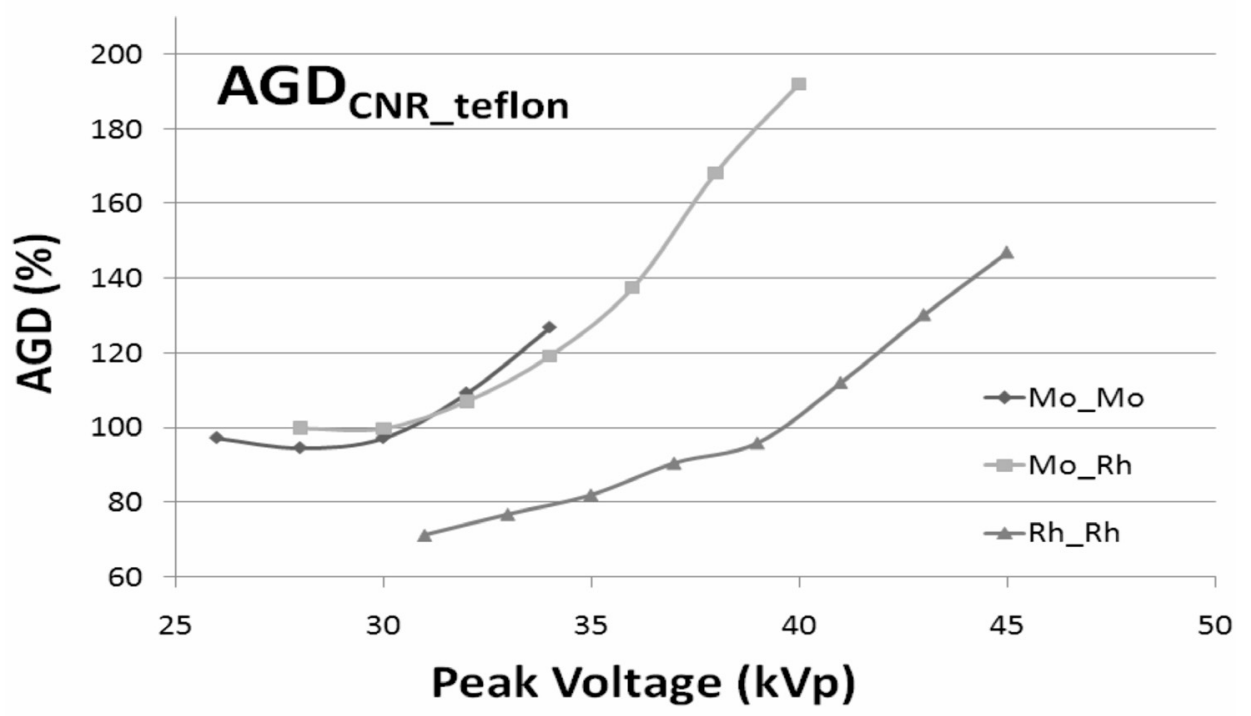

Figure 3. Relative AGD if high contrast CNR is to remain constant for different $\mathrm{kVp}$ and target filter combinations. The graphs are normalized to $28 \mathrm{kVp}$ for $\mathrm{Mo} / \mathrm{Rh}$ 


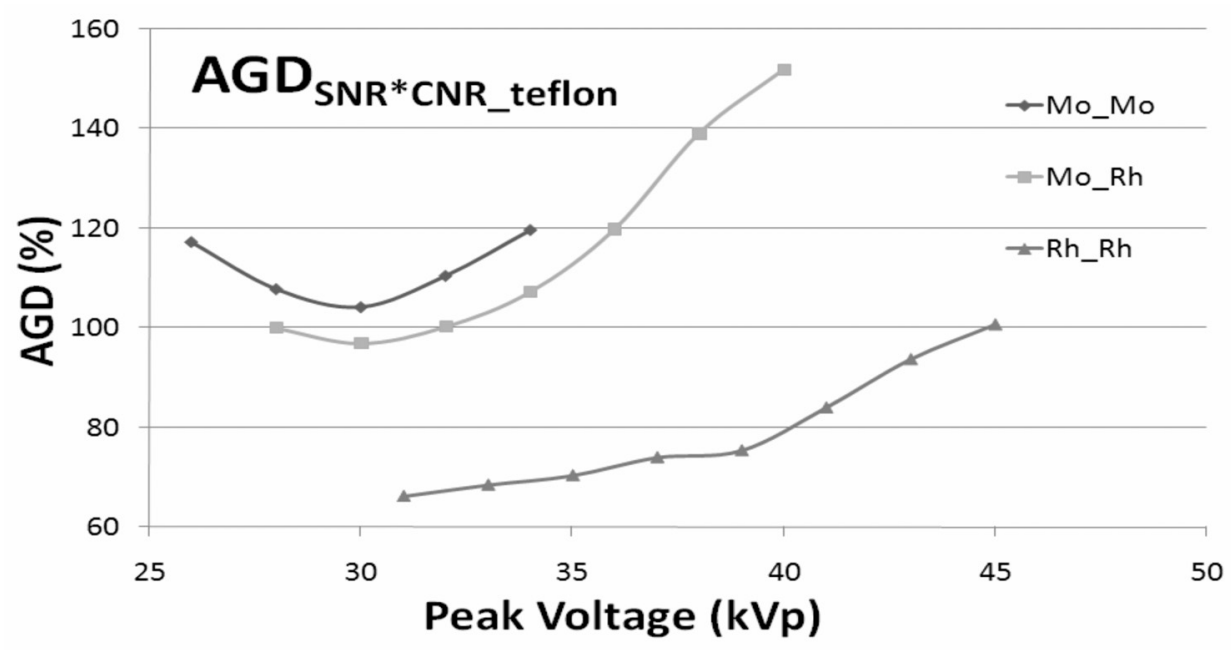

Figure 4. Relative AGD if the product of SNR and high contrast CNR is to remain constant for different $\mathrm{kVp}$ and target filter combinations. The graphs are normalized to $28 \mathrm{kVp}$ for $\mathrm{Mo} / \mathrm{Rh}$

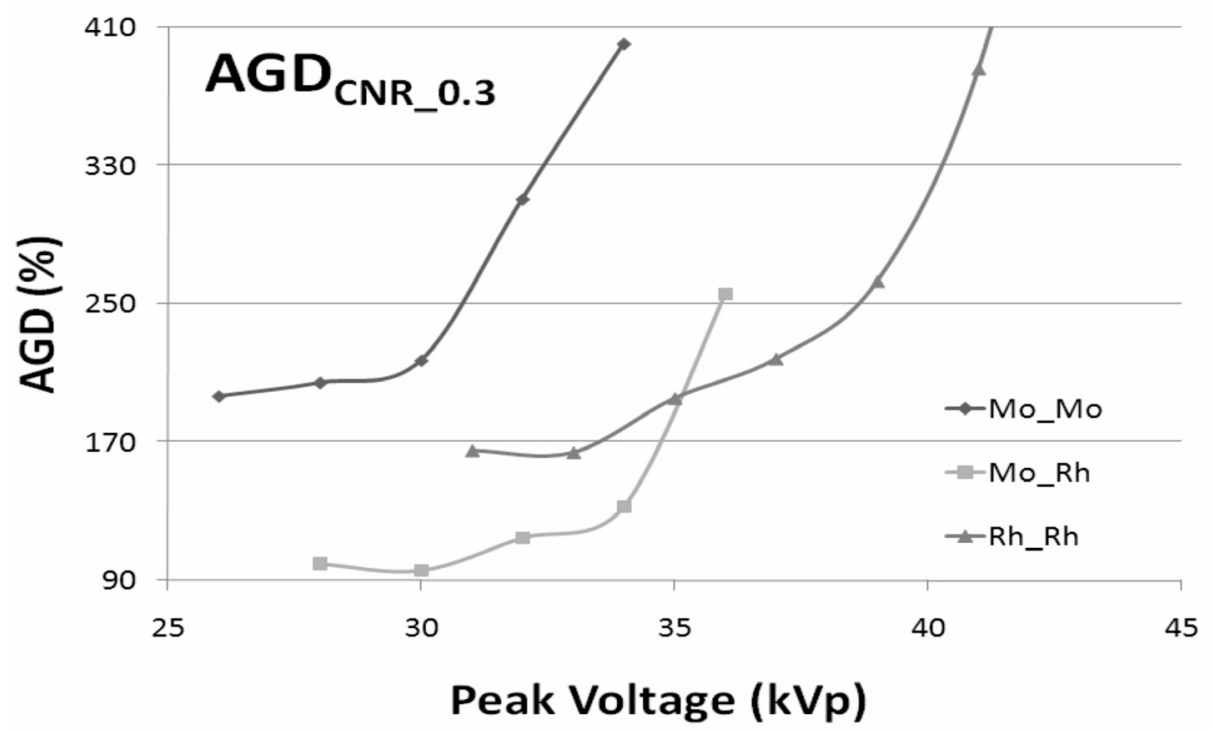

Figure 5. Relative AGD for low contrast CNR (cf. Figure 3). The graphs are normalized to $28 \mathrm{kVp}$ for $\mathrm{Mo} / \mathrm{Rh}$ 
Figures 2 through 4 present clear options for dose reduction of up to $40 \%$ compared to the stated standard techniques (SFM and FFDM), yet they only deal with pure SNR and high contrast resolution.

In contrast, Figure 5 shows low contrast resolution as the limiting factor in possible dose reduction. If the low contrast CNR values reached with the current standard setting of $28 \mathrm{kVp}$ for $\mathrm{Mo} / \mathrm{Rh}$ combination (Senographe 2000D) were to be the minimum required in clinical practice, no dose reduction would be possible. On the contrary, almost all changes in peak voltage and target/filter combination would demand a higher breast dose if the low contrast CNR is to be kept. It should particularly be noted, that switching to $\mathrm{Rh} / \mathrm{Rh}$ would require a substantial increase of dose to compensate for the decrease in the attenuation gradient for the harder X-ray spectrum produced using this target/filter combination.

It could be argued, that a clinically more realistic evaluation is given in Figure 6 where image quality is benchmarked by the product of SNR and CNR. This represents more or less the daily clinical trade-off between contrast and spatial resolution. However, even here no substantial dose reduction is possible, neither by increasing $\mathrm{kVp}$ nor by switching to other target/filter combinations.

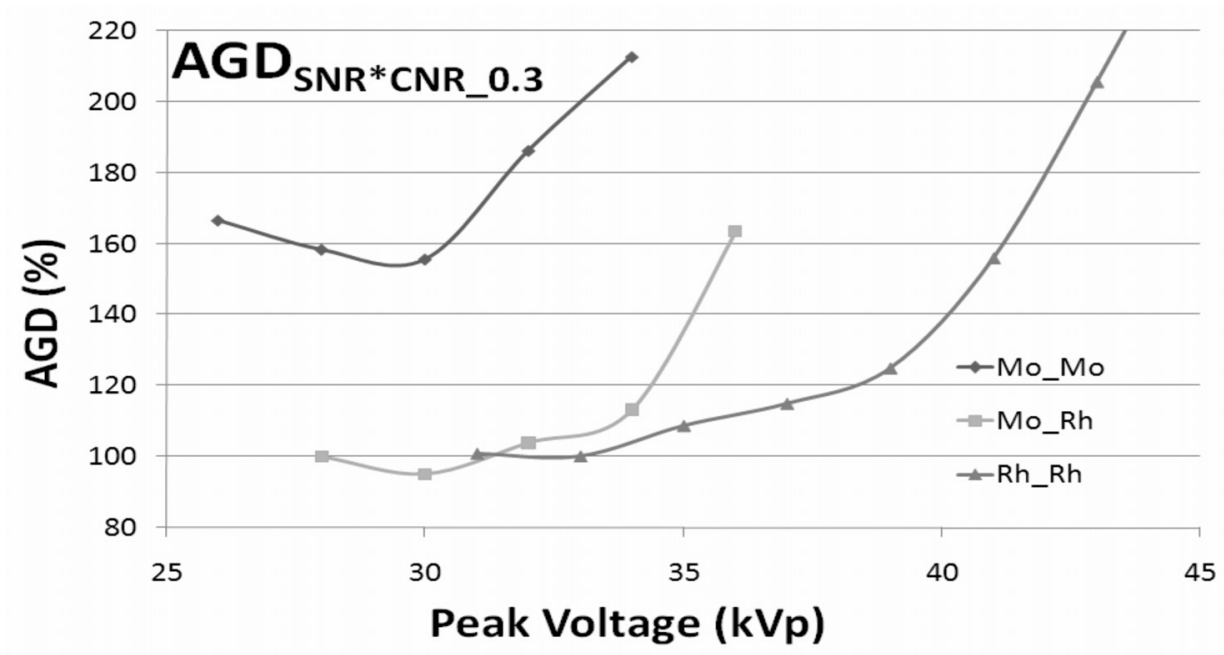

Figure 6. Relative AGD for constant product of SNR and low contrast CNR (cf. Figure 4). The graphs are normalized to $28 \mathrm{kVp}$ for Mo/Rh 


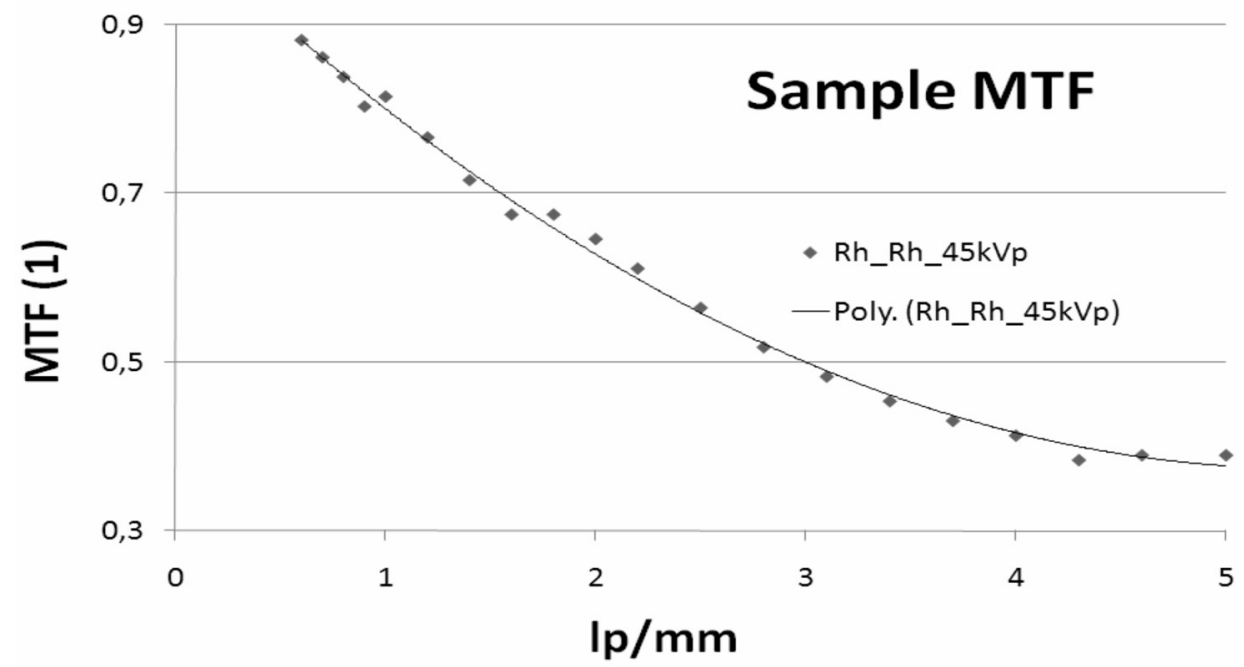

Figure 7. The MTF of the phantom image taken with a Rh/Rh combination at $45 \mathrm{kVp}$ and 100 mAs. All other MTF practically exhibit the same graph (cf. Figure 8)

Selected MTF values

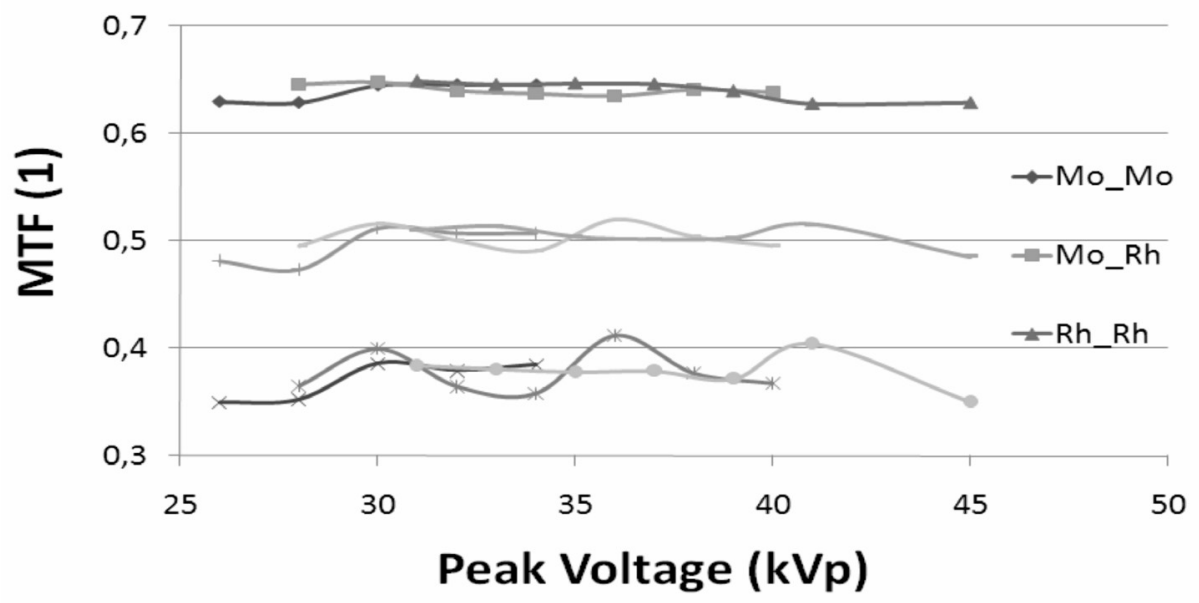

Figure 8. MTF values for $2.2 \mathrm{lp} / \mathrm{mm}, 3.1 \mathrm{lp} / \mathrm{mm}$, and $5.0 \mathrm{lp} / \mathrm{mm}$ taken for all measured phantom images. There is practically no difference between MTF's for different peak voltages and target/filter combinations 
Figures 7 and 8 show the MTF representing spatial resolution completely uncritical towards parameter change. This was expected since spatial resolution for digital systems is mainly limited by detector resolution (here about $100 \mu \mathrm{m}$ corresponding to $5 \mathrm{lp} / \mathrm{mm}$ ) kicking in way before any contrast problems could deteriorate the MTF.

This study shows that there is no potential in dose reduction if low contrast CNR levels are to be kept at those rendered by standard settings. In this case the digital system delivers at least around 2 mGy AGD what is in the range of conventional systems. For thicker breasts, the effect is not expected to change dramatically. Even if penetrability of harder X-ray spectra will improve the results slightly, it will not reverse them.

However, the linearity of detector response in conjunction with image post processing should allow for decreased CNR and hence AGD values. Partly, this assumption is already being implemented in the automatic mode of FFDM systems. For the Senographe 2000D, the DOSE mode ( $\mathrm{Rh} / \mathrm{Rh}$ at $31 \mathrm{kVp}$ ) delivers according to our measurements about $1.8 \mathrm{mGy}$ AGD for $4.5 \mathrm{~mm}$ breast thickness, i.e a 15\% dose reduction. However, a thorough study to test potential as well as the limits of this approach should be implemented before introducing it into clinical routine.

\section{Appendix}

The MTF is described as the ratio of the maximum amplitude for a signal with the frequency $f$ to the maximum amplitude to the zero frequency signal:

$$
\mathrm{MTF}=\frac{A_{\max }(f)}{A_{\max }(0)}
$$

For a pure sine signal it holds

$$
A_{\max }=C\left(\sin \frac{\pi}{2}-\sin \frac{3 \pi}{2}\right)=2 C
$$

The standard deviation $\sigma$ for this signal is

$$
\begin{aligned}
\sigma & =\sqrt{\frac{\sum_{i=1}^{n}\left(C \sin x_{i}-C \overline{\sin x_{i}}\right)^{2}}{n-1}} \\
& =C \sqrt{\frac{\sum_{i=1}^{n} \sin ^{2} x_{i}}{n-1}}
\end{aligned}
$$


with the average of $\sin \left(x_{i}\right)$ for full cycles equal to zero. For $m$ full cycles it now holds:

$$
\begin{aligned}
\sigma^{2} & =\frac{C^{2}}{n-1} \sum_{i=1}^{n} \sin ^{2} x_{i} \\
& \approx \frac{C^{2}}{2 m \pi} \int_{0}^{2 m \pi} \sin ^{2} x d x \\
& \approx \frac{C^{2}}{2}
\end{aligned}
$$

That leads to:

$$
\begin{aligned}
A_{\max } & =2 C=2 \sqrt{2} \sigma=\sqrt{8} \sigma \\
& \approx 2.8 \sigma
\end{aligned}
$$

i.e. the maximum amplitude of a full cycle sin wave is equal to the square root of eight times its standard deviation. This derivation holds true for two dimensions as long as the 2-D function can be described as a product of sin function as given above and a constant (as given for bar patterns where the constant is unity).

For a simple step function (true image of a bar pattern) one can analogously show that $A_{\max }$ is $2 \mathrm{C}$ and $\sigma$ is C, i.e. $A_{\max }=2 \sigma$. Since the real image of a bar pattern is superposition of step function and sin wave it holds in a sufficient approximation:

$$
\mathrm{MTF}=\frac{k \cdot \sigma(f)}{\left|M_{b g}-M_{\text {bar pattern }}\right|}
$$

with $M_{b g}$ and $M_{b a r}$ pattern as pixel values of the back ground and (unmodulated) bar pattern's high attenuating material. The factor $k$ (being the same for all measured curves) can be determined by extrapolating all measured MTF curves so that they render MTF $=1$ at $0 \mathrm{lp} / \mathrm{mm}$. The results should be within a few percent correspondence, in our case within $=1.5 \%$ for $k=2.2$.

\section{References}

[1] American College of Radiology, Mammography Quality Control Manual. American College of Radiology, Reston, VA, 1999

[2] Young KC. Radiation doses in the UK trial of breast screening in women aged 40-48 years. Br J Radiol. 2002; 75: 362-370. 
[3] Chevalier M, Moran P, Ten J, Soto J, Cepeda T, Vano E. Patient dose in digital mammography. Med Phys. 2004; 31(9): 2471-2479.

[4] Obenauer S, Hermann KP, Schorn C, Fischer U, Grabbe E. Full-field digital mammography: dose-dependent detectability of simulated breast lesions. Fortschr Rontgenstr. 2000; 172: 1052-1056.

[5] Kruger RL, Schueler BA. A survey of clinical factors and patient dose in mammography. Med Phys. 2001; 28(7): 1449-1454.

[6] Obenauer S, Hermann KP, Grabbe E. Dose reduction in full-field digital mammography: an anthropomorphic breast phantom study. Br J Radiol. 2003; 76: 478-482. 